\title{
Large $x$ Behaviour and the Non-Perturbative Structure of Hadronic Systems
}

\author{
Anthony W. Thomas \\ Jefferson Lab, 12000 Jefferson Ave., Newport News VA 23606, USA
}

\begin{abstract}
While the traditional interest in structure functions has been the confirmation of the predictions of perturbative QCD, this data also contains a wealth of information on how QCD works in the infrared, or confinement, region. As the challenge of the strong force now turns to the study of QCD in the non-perturbative region, such information is extremely valuable. We outline some of the key issues for both nucleon and nuclear structure functions.
\end{abstract}

\section{INTRODUCTION}

While considerable progress has been made towards understanding hadron structure, it is fair to remark that there are far more questions which remain unanswered than resolved. Amongst the open questions which go to the heart of how QCD is realized we mention:

- Does pQCD describe the behaviour of the $d / u$ ratio as $x \rightarrow 1[1,2,3]$ ?

- What role do di-quark correlations play in the spin and flavour dependence of the parton distribution functions (PDFs) [4]?

- What is the behaviour of the ratio $\Delta d / \Delta u$ as $x \rightarrow 1$ ?

- Is charge symmetry satisfied by the valence PDFs $[5,6]$ ? If not, how much of the NuTeV anomaly [7] does this explain [8]?

- Is the excess of $\bar{d}$ over $\bar{u}$ quarks in the proton [9] entirely explained by the spontaneous breaking of chiral symmetry $[10,11]$ ?

- Is there a similar violation [12] of the naive expectation that $\Delta \bar{u}=\Delta \bar{d}$ ? What is its source?

- Does the strange sea satisfy $\bar{s}=s$, or is this symmetry also violated by spontaneous chiral symmetry breaking [13] or some other mechanism?

- What is the role of heavier flavours in the nucleon - is there significant intrinsic charm/beauty [14]?

- Can the modern, rigorous version of the Ellis-Jaffe sum rule [15] be tested with precision?

- Does the Gottfried sum rule exist [16]?

- Can modern experiments improve the precision with which fundamental sum rules such as Gross-Llewellyn-Smith (currently 10-15\% precision) and Adler (currently $20-30 \%$ precision) are tested [17]? 
- To what extent is $S U(3)$ symmetry satisfied in the PDFs of the other octet baryons [18]?

- Can we relate baryon fragmentation functions to the PDFs of the baryons [19] - in the valence region.... in the sea region?

- What is the origin of the famous, nuclear EMC effect [20] in the valence region?

- Can we differentiate between the effects of short-range correlations, multi-quark states and other potential contributions to nuclear structure functions in the kinematic region $x>1$ ?

- What is the contribution of polarized glue in the proton spin structure function [21]?

- Are we able to reliably extract higher twist contributions to free nucleon structure functions [22]?

- What is the role of shadowing in existing nuclear structure functions? Is there a significant higher twist component and what role do differences between higher twist components in electromagnetic, charged and neutral current neutrino scattering [23] play in the NuTeV anomaly [24]?

- What is the role of Lorentz scalar and vector mean fields in atomic nuclei in modifying the nuclear PDFs $[25,26]$ ? Are nuclear PDFs related in any simple way, as usually assumed, to the PDFs of the free nucleon?

- Given recent advances in lattice QCD are we able to contribute anything helpful from that source?

To be honest, after compiling this list of questions I was astonished. All are real and all are important and there are a lot of them! Resolving them is an outstanding challenge in hadronic physics for the next decade, experimentally and theoretically. It is certainly a major part of the mission of Jefferson Lab at $12 \mathrm{GeV}$ and beyond. Apart from listing them here with minimal references for those interested to at least get an introduction to the issues, we cannot explore more than couple of topics.

\section{LATTICE QCD}

The problems faced in performing lattice QCD simulations at light quark mass are relatively well known, with the time for a calculation scaling as roughly $m_{\pi}^{7}$ or worse [27]. In addition, because lattice simulations are performed in Euclidean space, one can only access information on PDFs through their moments. Furthermore, problems of operator mixing associated with the $\mathrm{H}(4)$ symmetry of a lattice, mean that only low moments are currently accessible - up to $x^{3}$ for unpolarised moments and $x^{2}$ in the spin dependent case. Since the large- $x$ behaviour of the PDFs is determined by the higher moments, it is not likely that one will unambiguously fix their behaviour in that region from lattice QCD. Nevertheless it is of great interest to see exactly what one can learn from such calculations.

The outstanding problem in the field for some years was a continuing failure of the lattice simulations to reproduce the momentum fraction of the nucleon carried by its valence quarks. In particular, the integral of the non-singlet combination $u_{V}-d_{V}$, weighted by $x$, was typically a factor of two larger than experiment. For a very fundamental quan- 


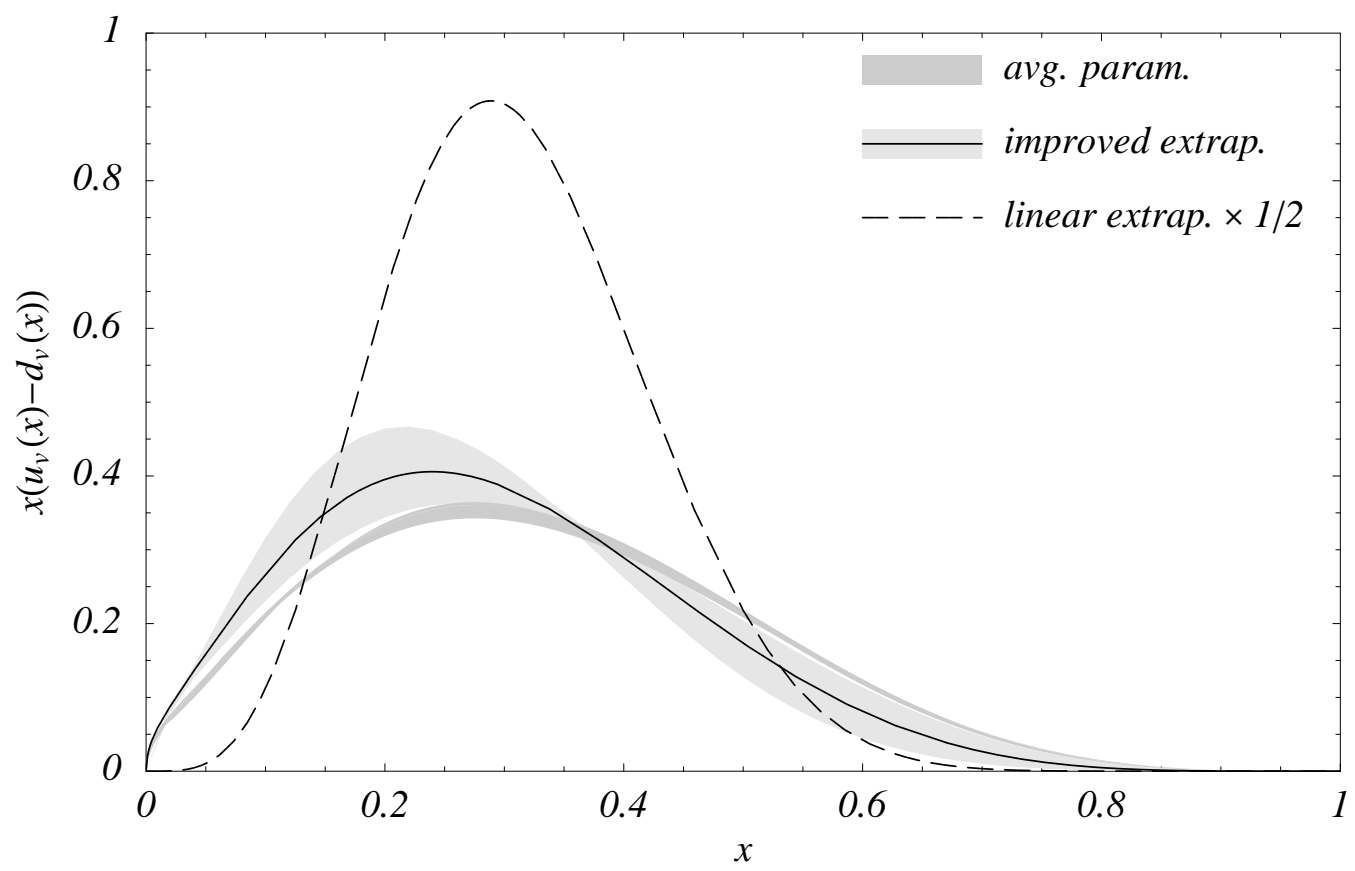

FIGURE 1. Comparison between the isovector valence quark distribution of the proton reconstructed from the lattice moments after a correct chiral extrapolation (solid curve and light shaded area) with the commonly used parametrizations of world data (dark shaded area). The dash-dot curve shows the result if the extrapolation of the moments is made with a simple linear fit - from Ref. [29].

tity which characterizes the sharing of momentum between quarks and gluons in the proton, this was an intriguing problem. Only in 2001 was it realized that because the calculation of PDFs involves a forward matrix element, chiral corrections can be extremely important and indeed the chiral logarithm associated with this problematic moment is expected to lead to a rapid variation as $m_{\pi}$ approaches its physical value. Taking this into account removed the long-standing discrepancy [28].

Having realized the importance of a correct chiral extrapolation of the moments it is possible to parametrize all the moments as a function of pion mass. Then by assuming a particular functional form for the PDF one can determine the parameters of the phenomenological function which provide a best fit to the lattice data at each pion mass. This approach necessarily involves a degree of model dependence in any conclusions drawn, but this seems unavoidable. Nevertheless, as we see in Fig. 1, the level of agreement between the PDF extracted from such a fitting process, using moments extrapolated with the correct chiral behaviour to the physical mass, and experiment seems very good [29]. The lack of higher moments, as well as the statistical errors in the lattice moments and, of course, uncertainty in the chiral extrapolation, lead to a relatively large error band on the theoretical curve. A similar study has been carried out for the spin dependent PDFs, with necessarily larger errors as a consequence of having one less moment in that case [36].

Another feature of Fig. 1 that requires attention is the reconstructed valence PDF at a current quark mass above $50 \mathrm{MeV}$. While this does not seem so much larger than 


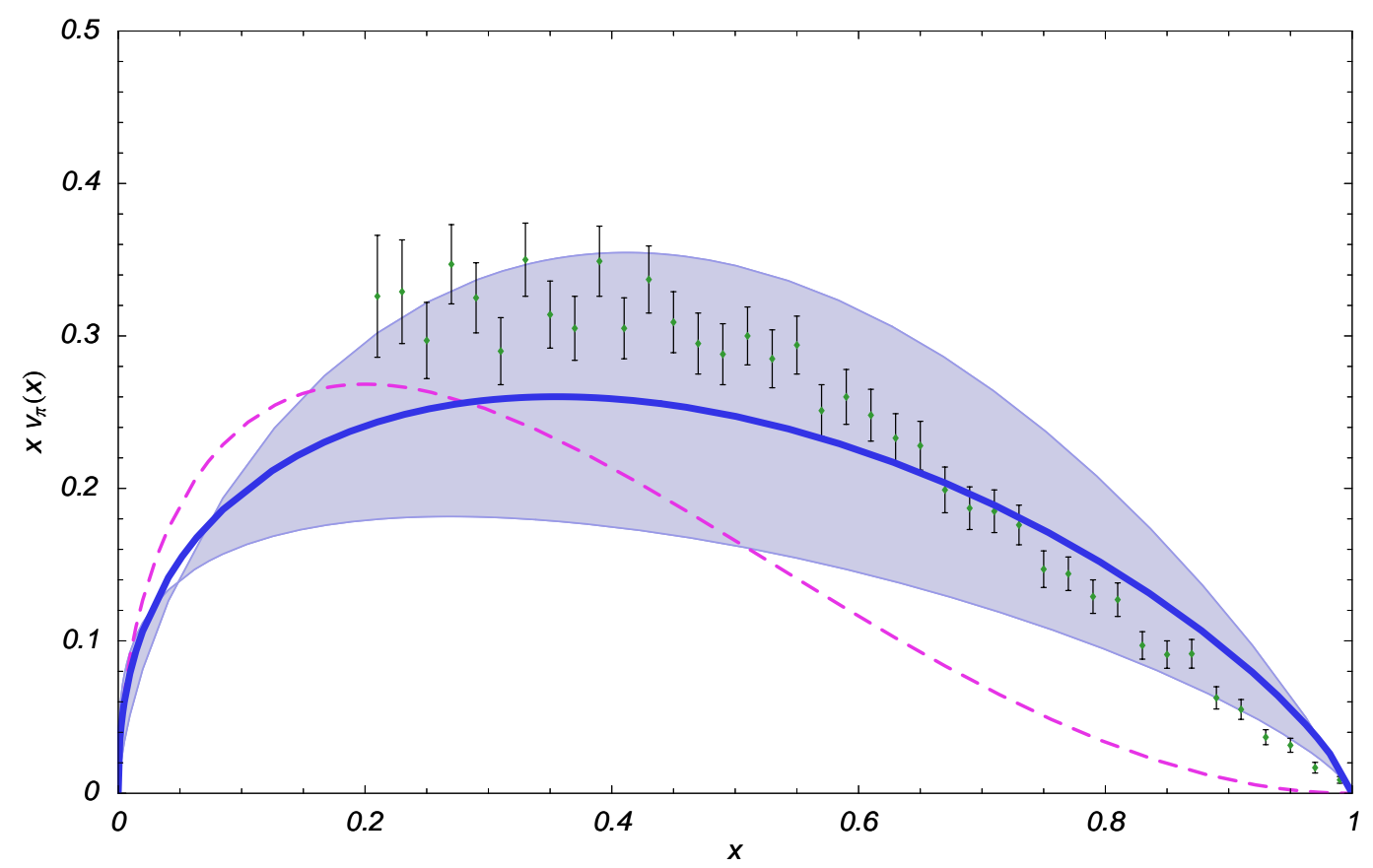

FIGURE 2. Comparison between Drell-Yan data for the valence PDF of the pion and the reconstruction based on a chiral extrapolation of lattice moments (solid line and shaded area). For comparsion the commonly used from based on pQCD is shown as the dashed curve - from Ref. [36].

the physical quark mass the physical difference is enormous. At this point the pion mass is sufficiently large that its Compton wavelength is smaller than the source of the pion field and, as a consequence, pion loops are almost totally suppressed [30]. As a corollary the constituent quark model seems to suddenly describe the structure of the nucleon extremely well. Indeed, we see that the PDF clearly peaks around $1 / 3$, with the three valence quarks sharing the momentum of the nucleon. This strongly supports other evidence based on the behaviour of masses, magnetic moments and charge radii calculated on the lattice, which suggests that this slightly unphysical mass regime is the best place to build a constituent quark model [31].

While much attention has been focussed on the nucleon, perhaps because of the amount of experimental information we have on its PDFs, the pion is also of great interest. The Drell-Yan data, which have yielded much of our information on its large$x$ structure $[32,33]$, seem to contradict the perturbative QCD (pQCD) expectation that the valence distribution should go as $(1-x)^{2}$ as $x \rightarrow 1$ [34]. It is therefore of great interest to see whether lattice QCD can give any information [35]. Using the same technique described earlier for the nucleon, to reconstruct the valence PDF of the pion is even more challenging - there are less moments and they alternate (for even and odd moments) between singlet and non-singlet. However, using the fact that chiral loops are suppressed at pion masses above $0.4-0.5 \mathrm{GeV}$, Detmold et al. were able to obtain the distribution shown in Fig. 2 [36]. The errors are clearly large and without more modern data (and ideally more moments) one cannot make a firm conclusion. Nevertheless, it is clear that the calculated PDF does agree with the experimental data and furthermore that 
it does tend to (slightly) favour a harder distribution than pQCD would suggest. Further progress awaits new and more precise data.

\section{CONCLUDING REMARKS}

We have seen that in terms of fundamental calculations based on non-perturbative QCD, there has been considerable progress in extracting PDFs for the nucleon and pion. For the moment this process is model dependent and the accuracy is limited by the small number of moments that can be calculated and the relatively large quark masses for which data is available. In the near future we can expect the rapidly improving computer power to yield partially quenched or, better, full QCD data at masses nearer the physical regime. In addition, group theoretical work as well as improved algorithms will enable us to calculate more moments and hence better constrain the large- $x$ behaviour.

On the other hand, when it comes to the important issues surrounding nuclear PDFs, where in fact much of our information about PDFs has been obtained, lattice QCD is unlikely to be of any help in the forseeable future. We have no choice but to develop more sophisticated models of nuclear structure starting at the quark and gluon level, which respect the constraints of special relativity [37]. In particular, we are going to have to rely on such models, as well as new experimental data and good phenomenology, in order to answer the fundamental question whether there is indeed any simple relation between nucleon and nuclear structure functions. Certainly the recent studies of the different roles of scalar and vector mean fields in models of the QMC type [25, 26], suggest that there may well be significant differences and this would be of considerable importance.

\section{ACKNOWLEDGMENTS}

I would like to particularly acknowledge the contributions of Wolfgang Bentz, Will Detmold, Wally Melnitchouk, Fernando Steffens and Kazuo Tsushima to the main topics discussed here. This work was supported by DOE contract DE-AC05-84ER40150, under which SURA operates Jefferson Lab.

\section{REFERENCES}

1. G. R. Farrar and D. R. Jackson, Phys. Rev. Lett. 35, 1416 (1975).

2. W. Melnitchouk and A. W. Thomas, Phys. Lett. B 377, 11 (1996) [arXiv:nucl-th/9602038].

3. S. J. Brodsky, M. Burkardt and I. Schmidt, Nucl. Phys. B 441, 197 (1995) [arXiv:hep-ph/9401328].

4. F. E. Close and A. W. Thomas, Phys. Lett. B 212, 227 (1988).

5. E. Sather, Phys. Lett. B 274, 433 (1992).

6. E. N. Rodionov, A. W. Thomas and J. T. Londergan, Mod. Phys. Lett. A 9, 1799 (1994).

7. G. P. Zeller et al. [NuTeV Collaboration], Phys. Rev. Lett. 88, 091802 (2002) [Erratum-ibid. 90, 239902 (2003)] [arXiv:hep-ex/0110059].

8. J. T. Londergan and A. W. Thomas, Phys. Rev. D 67, 111901 (2003) [arXiv:hep-ph/0303155].

9. P. Amaudruz et al. [New Muon Collaboration], Phys. Rev. Lett. 66, 2712 (1991). 
10. A. W. Thomas, Phys. Lett. B 126, 97 (1983).

11. D. B. Leinweber et al., Phys. Rev. Lett. 86, 5011 (2001) [arXiv:hep-ph/0101211].

12. A. W. Schreiber, A. I. Signal and A. W. Thomas, Phys. Rev. D 44, 2653 (1991).

13. A. I. Signal and A. W. Thomas, Phys. Lett. B 191, 205 (1987).

14. S. J. Brodsky, P. Hoyer, C. Peterson and N. Sakai, Phys. Lett. B 93, 451 (1980).

15. S. D. Bass et al., Phys. Rev. D 66, 031901 (2002) [arXiv:hep-ph/0207071].

16. V. Guzey et al., Phys. Rev. D 64, 054503 (2001) [arXiv:hep-ph/0102133].

17. A. W. Thomas and W. Weise, "The Structure of the Nucleon," Wiley-VCH, Berlin, 2001.

18. M. Alberg et al., Phys. Lett. B 389, 367 (1996) [arXiv:hep-ph/9609498].

19. C. Boros et al., Phys. Rev. D 62, 014021 (2000) [arXiv:hep-ph/0002081].

20. J. J. Aubert et al. [European Muon Collaboration], Phys. Lett. B 123, 275 (1983).

21. J. Ashman et al. [European Muon Collaboration], Phys. Lett. B 206, 364 (1988).

22. Z. E. Meziani et al., arXiv:hep-ph/0404066.

23. C. Boros et al., Phys. Rev. D 59, 074021 (1999) [arXiv:hep-ph/9810220].

24. G. A. Miller and A. W. Thomas, arXiv:hep-ex/0204007.

25. H. Mineo et al., Nucl. Phys. A 735, 482 (2004) [arXiv:nucl-th/0312097].

26. F. M. Steffens et al., Phys. Lett. B 595, 237 (2004) [arXiv:hep-ph/0405096].

27. T. Lippert, S. Gusken and K. Schilling, Nucl. Phys. Proc. Suppl. 83, 182 (2000).

28. W. Detmold et al., Phys. Rev. Lett. 87, 172001 (2001) [arXiv:hep-lat/0103006].

29. W. Detmold et al., Mod. Phys. Lett. A 18, 2681 (2003) [arXiv:hep-lat/0310003].

30. W. Detmold et al., Pramana 57, 251 (2001) [arXiv:nucl-th/0104043].

31. I. C. Cloet et al., Phys. Rev. C 65, 062201 (2002) [arXiv:hep-ph/0203023].

32. J. Badier et al. [NA3 Collaboration], Z. Phys. C 18, 281 (1983).

33. B. Betev et al. [NA10 Collaboration], Z. Phys. C 28, 15 (1985).

34. G. P. Lepage and S. J. Brodsky, Phys. Rev. D 22, 2157 (1980).

35. G. Martinelli and C. T. Sachrajda, Nucl. Phys. B 306, 865 (1988).

36. W. Detmold et al., Phys. Rev. D 68, 034025 (2003) [arXiv:hep-lat/0303015].

37. P. A. M. Guichon and A. W. Thomas, Phys. Rev. Lett. 93, 132502 (2004) [arXiv:nucl-th/0402064]. 